\title{
Looking Back is Looking Forward: The Need for Retrospective Solar System Observations in Advance of Exoplanet Retrievals
}

Chester "Sonny" Harman"1, Noam R. Izenberg², Kevin B. Stevenson², Michael Zemcov ${ }^{3}$, Carey M. Lisse ${ }^{2}$, Giada Arney ${ }^{4}$, Seth Redfield ${ }^{5}$, Edward Schwieterman 6 , Abigail Rymer ${ }^{2}$, Andrew Poppe ${ }^{7}$, Kathleen E. Mandt ${ }^{2}$

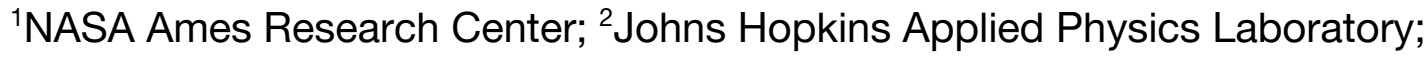
${ }^{3}$ Rochester Institute of Technology; ${ }^{4}$ NASA Goddard Space Flight Center; ${ }^{5}$ Wesleyan University; ${ }^{6}$ University of California, Riverside; ${ }^{7}$ University of California, Berkeley; †(650-604-1671; sonny.harman@nasa.gov)

\section{Plain language summary}

Looking back at the solar system offers several unique scientific and social perspectives, largely captured in a piecemeal fashion during past missions. These observations and the hypotheses based on them can be verified with other techniques and missions, unlike for extrasolar planets, where no such corroborating data sets are reasonably expected. This makes analog data sets, specifically observations of the solar system at larger distances, a critical stepping stone towards reliably interpreting future exoplanet observations. Looking back at the solar system should be encouraged for future missions by explicitly listing it as a target of opportunity.

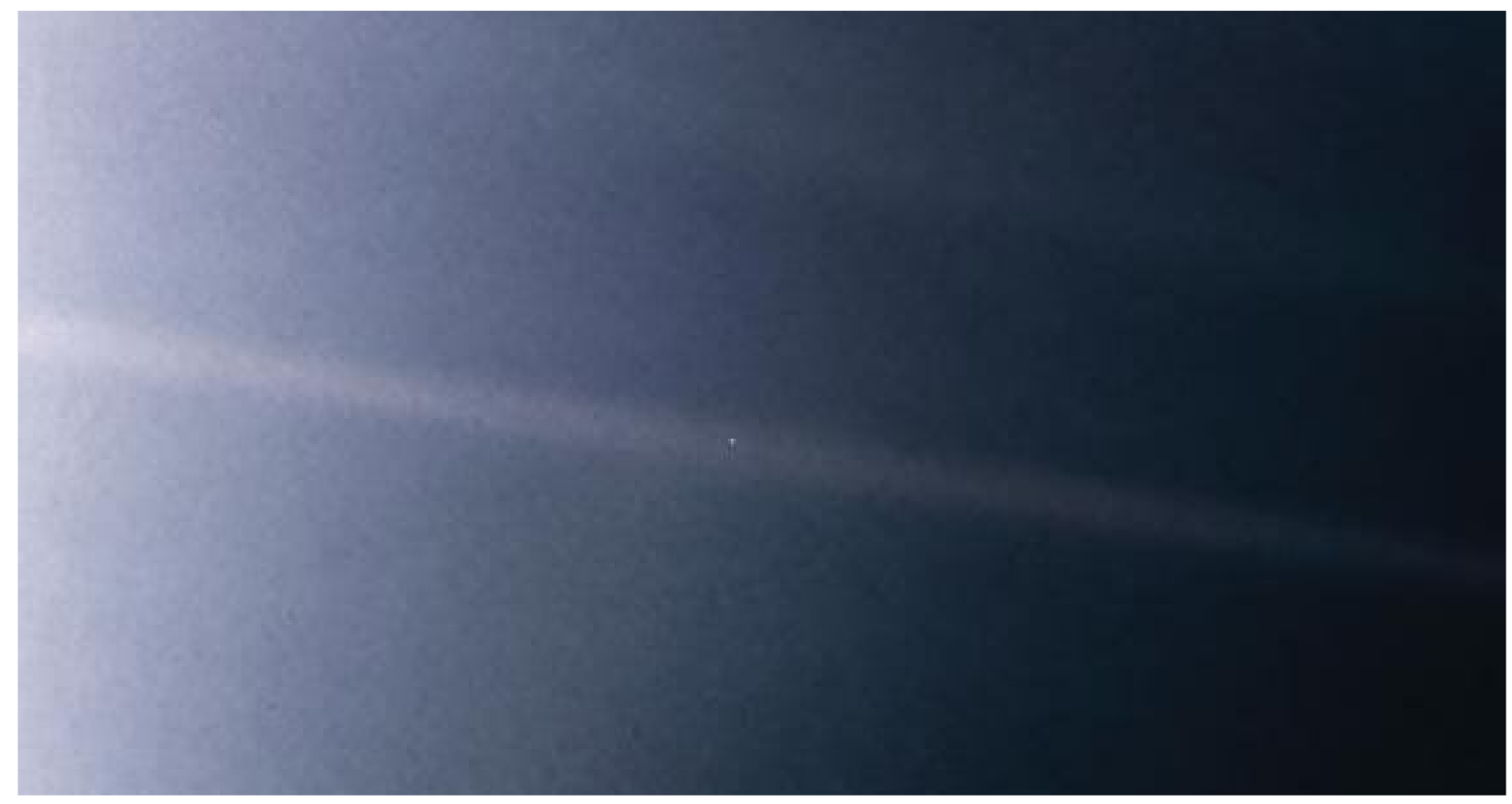

PIA23645: Pale Blue Dot Revisited (Gill, Hansen, and Kosmann; NASA/JPL-CalTech) 


\section{Introduction}

We advocate here for the intentional inclusion of retrospective observations of the solar system as distinct targets of opportunity for all future space missions. When missions have made observations of other objects outside the scope of their primary mission, they have shed light on a range of phenomena, from the nature of planetary atmospheres [e.g., 1,2] and surfaces [e.g., 3,4] to the impact of life on the Earth's integrated spectrum $[5,6]$. Often, these observations are made when the spacecraft is close to the target of opportunity, but on rare occasions, measurements $[7,8]$ and images (e.g., Pale Blue Dot) have been taken at large separations. Such 'look-back' opportunities are unique and gripping, with broad scientific and social ramifications that foster novel research and engage the public in enduring ways.

Scientifically, look-back observations can provide normally inaccessible viewing geometries and conditions (e.g., both low and high phase angles of Venus are inaccessible from Earth [9]), as well as potentially novel observational modes (such as at unique wavelength ranges [10]). These data sets also serve as a bridge between planetary and exoplanetary science, effectively linking ground truth to observations analogous to those we might make of the nearest exoplanets [see also 11,12]. Such observations are not limited to planets; for example, zodiacal dust impacts future observations of exoplanets [13], and extragalactic background light is difficult to measure through it [14]. Importantly, many of the most culturally significant images from space have been taken without an immediate scientific objective (for example, Earthrise). We outline here the unique science and engagement opportunities that look-back observations can simultaneously provide for planetary and Earth science, astrobiology, heliophysics, and astrophysics (underscoring the need for crossdisciplinary direction [15]), closing with an example using the Interstellar Probe [16].

\section{Science from Retrospective Observations}

Phase, Variability, and Unique Viewing Geometries

There is a constantly growing body of remote sensing observations of solar system objects from ground- and space-based platforms. These data sets are invaluable assets in validating forward retrieval models of what we can expect to see for some exoplanets, as well as a solid foundation for extrapolation. While this data is largely available, there are still barriers between exoplanetary and planetary science that must be addressed [11]. Here we highlight several examples of novel insights from solar system missions, but also point to where the lack of planetary and solar data has direct implications for accurately interpreting observations of exoplanetary systems.

The Earth is the only confirmed habitable and inhabited planet at this time. Spectroscopic observations of the atmosphere capture direct indicators of habitability and life, such as water vapor, oxygen, and the vegetation red edge, as well as indirect 
proxies like Earth's surface dichotomy [17]. Detecting glint (specular reflection off of the ocean's surface) has been suggested as one possible way to directly detect liquid water [5], which has been borne out by observations [4]. Much like water vapor, the detection of glint by itself is strongly suggestive of, but does not guarantee, liquid water, given other liquids (e.g., Titan [18]), surface ambiguities [19], or other forward-scattering effects [4]. The glint effect is strongest at high phase angles because of the sharp transition between absorption and reflection at the critical angle. Including it in models has a significant effect (up to 50\%) [20], much larger than the apparent brightness change induced by viewing geometry [21]. But whole-disk Earth observations at high phase angles are rare. For example, while the DSCOVR mission provides continuous monitoring of the Earth from the Sun-Earth L1 Lagrange point [e.g., 22], they are entirely at full phase (100\% illumination).

Beyond this, the Earth has a uniquely dynamic nature, visibly varying on the scale of hours, days, seasons, and years (setting aside decadal and longer timescales). Earth's axial tilt and the asymmetric distribution of continents modulate a strong biological modification of the atmosphere [e.g., 23]. Other events, like volcanic eruptions, wildfires, and synoptic-scale clouds and storms, occur fairly regularly, but not predictably, and can substantially modify the disk-integrated spectra of the Earth through the modification of trace gas amounts or by obscuring surface features. These types of events are difficult to intentionally capture, making the use of simplified phase curves and apparent magnitudes problematic [24,25]. This further emphasizes the critical need for dedicated space-based observations of the temporal evolution of Earth via disk-integrated observations [26].

Moving past Earth, there are similar outstanding questions for the other solar system planets with regards to phase-, geometry-, wavelength-, and time-dependent observations. As mentioned previously, Venus (and Mercury) are impossible to view at low or high phase angles from Earth [9], but this only scratches the surface. One consequence of Venus' cloud cover is an upturn at high phase angles caused by the clouds' enhanced forward scattering [24]. Phase dependent information combined with other information like polarization can provide key diagnostic information on aerosol properties: this was used to indicate definitively that Venus' clouds are composed of sulfuric acid [27]. Forward scattering and its impact on a planet's appearance is dependent on atmospheric structure and composition, and in turn, the wavelength of the observation matters. It is also not unique to Venus, and in the extreme (for example, for Saturn's moon, Titan), the back-illuminated planet can appear brighter than when fully-lit and face-on [28]. Farther out in the solar system, broadband phase curves for Jupiter are largely derived from Earth-based observations $(<12$ degrees), with some data from Cassini's flyby for higher phase angles ( $<130$ degrees), but are unconstrained beyond that [25]. The phase curves for Uranus, Saturn, and Neptune are 
similarly based on a single flyby (Pioneer for Saturn, and Voyager 2 for Uranus and Neptune); Saturn's rings have not been observed beyond a narrow phase angle of $\sim 6$ degrees (ibid.). Importantly, Jupiter's phase curve is neither constant in time, uniform across all wavelengths, nor well-captured by simplifying assumptions, such as treating it as a Lambertian surface or neglecting hazes and chromophores produced by photochemistry [29 and references therein]; this is also true for the other giants. Without observations of these worlds at larger phase angles and over time, we risk missing critical data that can answer questions relevant to the planetary, heliophysics, astronomy, and exoplanet communities.

\section{The Solar System as the Closest (Ground-Truthed) Exoplanet System}

Looking at the solar system planets from large separations remains the first opportunity at making observations analogous to those planned for exoplanetary systems. Critically, the solar system is the only collection of planetary objects for which we can independently verify remote sensing data with other observational techniques. Luckily, the solar system planets are a microcosm of possible exoplanet states [see also 30]. Core-dominated exoplanets that broadly resemble Mercury have been discovered [e.g., 31], but there are still questions about Mercury's (and thus Mercury-like exoplanets') formation and evolution that can only be addressed with further investigation [32]. Venus-like planets are generally going to be the easiest terrestrial exoplanets to detect and characterize, but distinguishing between early-Earth- and Venus-like planets may not be straightforward without a better understanding of Venus' evolution [33]. Stratosphere-skimming transit observations of exoVenus planets may also struggle to distinguish cloudy Venus-like planets with thick atmospheres from clear-sky planets with thin atmospheres stripped by stellar activity [34]. Venus-like planets also provide one of the only avenues to observationally constrain the far future of Earth in the face of an ever-brightening Sun, offering a potential constraint on planetary habitability. Earth itself has worn many faces throughout its history, with atmospheres of distinct compositional and climatic types [e.g., 35], all more accessible to direct examination than non-terrestrial locales. Mars, as arguably the most well-studied terrestrial planet beyond Earth, has shown that while continued scrutiny unveils some answers, it invariably opens the door to more questions [e.g., 36]. In turn, Mars can provide us a unique window into conditions in the early solar system, with implications for Earth's evolution that are largely inaccessible to terrestrial records [35]. The giant planets are the first to have their exoplanet cousins characterized, but the family resemblance is difficult to judge in the absence of more information about their formation, possible migration, and core composition, especially for the ice giants [e.g., 37]. 
There are also trends within the solar system that can inform exoplanet studies. For example, ongoing efforts to quickly categorize exoplanets using the broadband colors of the solar system planets (focusing on potentially habitable planets) has met with some success [e.g., 38]. Likewise, the aerosols present in the atmospheres of several solar system planets (and potentially more, during earlier epochs) can offer potential insights into the growing number of hazy exoplanets [e.g. 39]. While it is difficult to capture clouds and haze in a parametric fashion, explicitly modeling its effects has proved invaluable in quantifying the impact of aerosols [e.g., 40]. Further characterization of hazy solar system planets is another critical need. In order to effectively leverage the growing body of in-situ measurements of the solar system objects to serve as ground truth, it is imperative that exoplanetary and planetary science identify common goals, foster knowledge exchange, develop shared funding lines, and prioritize observations that can serve multiple purposes [15].

\section{Observational Hurdles as Additional Science Return and Progress Markers}

Aside from the knowledge gaps within planetary science that feed some of the uncertainties of exoplanetary studies (and vice versa), retrospective observations of the solar system from any platform would provide practical experience in dealing with what are expected to be common hurdles to exoplanet characterization, as well as useful science return regarding those same obstacles. For example, Venus and Mercury are likely to be most affected by small inner working angles, image saturation, or scattering of sunlight into a camera's optics than the other planets (for example, Voyager 1's "Family Portrait", which includes Pale Blue Dot), making them an excellent choice for field-testing cutting-edge observational tools and techniques necessary for exoplanet detection and characterization [41,42]. Overcoming these challenges is an effective practical demonstration of the requisite technology to characterize exoplanets. Further sources of noise, like intervening zodiacal dust native to the solar system, also introduces noise from within exoplanet systems [13]. Looking back at the inner solar system, then, could provide further insights into the nature of zodiacal dust as a function of time, or with different wavelength or spectral resolution coverage compared to prior observations, allowing for more accurate models of exo-zodiacal dust. Similarly, background objects, such as faint stars, free-floating planets, or distant Kuiper Belt objects, are potentially a source of both consternation and discovery. Accounting for and seeking out opportunities to catalog and characterize these objects can yield new scientific findings [e.g., 43,44]. The challenges posed to any one discipline's observations is a treasure trove of data in another field, reinforcing the need for agency-wide complementary approaches. 


\section{Why We Look Back - Our Cosmic Home in Context}

Even neglecting the wide-ranging scientific opportunities we've listed so far, the ability to look back and capture the Earth in turn captures the world's imagination. This social aspect has instilled a long-running, overwhelmingly positive public interest, typified by Pale Blue Dot and Earthrise [see also 45]. Retrospective observations add an approachable and understandable facet to any cutting-edge science payload. They also serve an important role in how we as a society contextualize our place in the universe, and epitomize NASA's vision: "to discover and expand knowledge for the benefit of humanity."

\section{An Example - the Interstellar Probe}

Nominally the Interstellar Probe (ISP) is a mission through the outer heliosphere and to the nearby "Very Local" interstellar medium (VLISM). ISP has been a mission of significant interest to the heliophysics community for some time [16,46], and the 2010 Heliophysics Decadal Survey calls for the future launch of the first dedicated ISP. Escaping the solar system's boundaries would enable unique views of the global heliosphere in the context of other astrospheres, further discoveries of unexplored Kuiper Belt Objects, and the first observations of our circumsolar dust disk. This naturally bridges the planetary, heliophysical, and astrophysical disciplines by putting our solar system and heliosphere in the context of the increasing number of detected and characterized exoplanetary systems and astrospheres.

The primary science target of ISP will be the particles, fields, and dust of the VLISM and their interaction with the heliosphere (likely necessitating a spinning spacecraft). That said, ISP's mission profile offers truly unique viewing geometries and observational timescales capable of filling fundamental knowledge gaps in planetary and exoplanetary science like the ones we have outlined above. The current notional trajectory will take ISP within 45 degrees of the "nose" (or apex) of the heliosphere's ram direction through the VLISM, in the plane of the ecliptic, at $~ 8$ au/year. ISP, being designed for a 50 year long generational mission, will travel 5 to 10 times farther away from Earth and the Sun than any previous mission.

\section{What Retrospective Observations from ISP Might Look Like}

Given ISP's notional mission profile and primary science drivers, buy-in from the planetary science community might take the form of one or more small instruments included in ISP's payload. One such option would be a $<500 \mathrm{~g}$ backward-facing cluster of straw-telescopes, fixed along the spacecraft-Sun line. These co-boresighted straw-telescopes could either feed a small spectrograph or have dedicated broadband detectors, chosen based on what would maximize science return. For example, visible and infrared channels could be used to build datasets capable of distinguishing 
between rocky and giant planets, while ultraviolet channels could detect Rayleigh scattering, ozone absorption, and upper atmospheric composition. Pointing the telescopes slightly off-axis allows for long-term, time-resolved monitoring of the planets of the inner solar system, and the outer planets as ISP moves farther away [see also 47], with the potential for full phase curves of all the planets out to (and including) Saturn (Fig. 1). Field of view and sensitivity considerations would form a natural trade space, customizable to which targets, and over what temporal scales and separations, are priorities for the community.
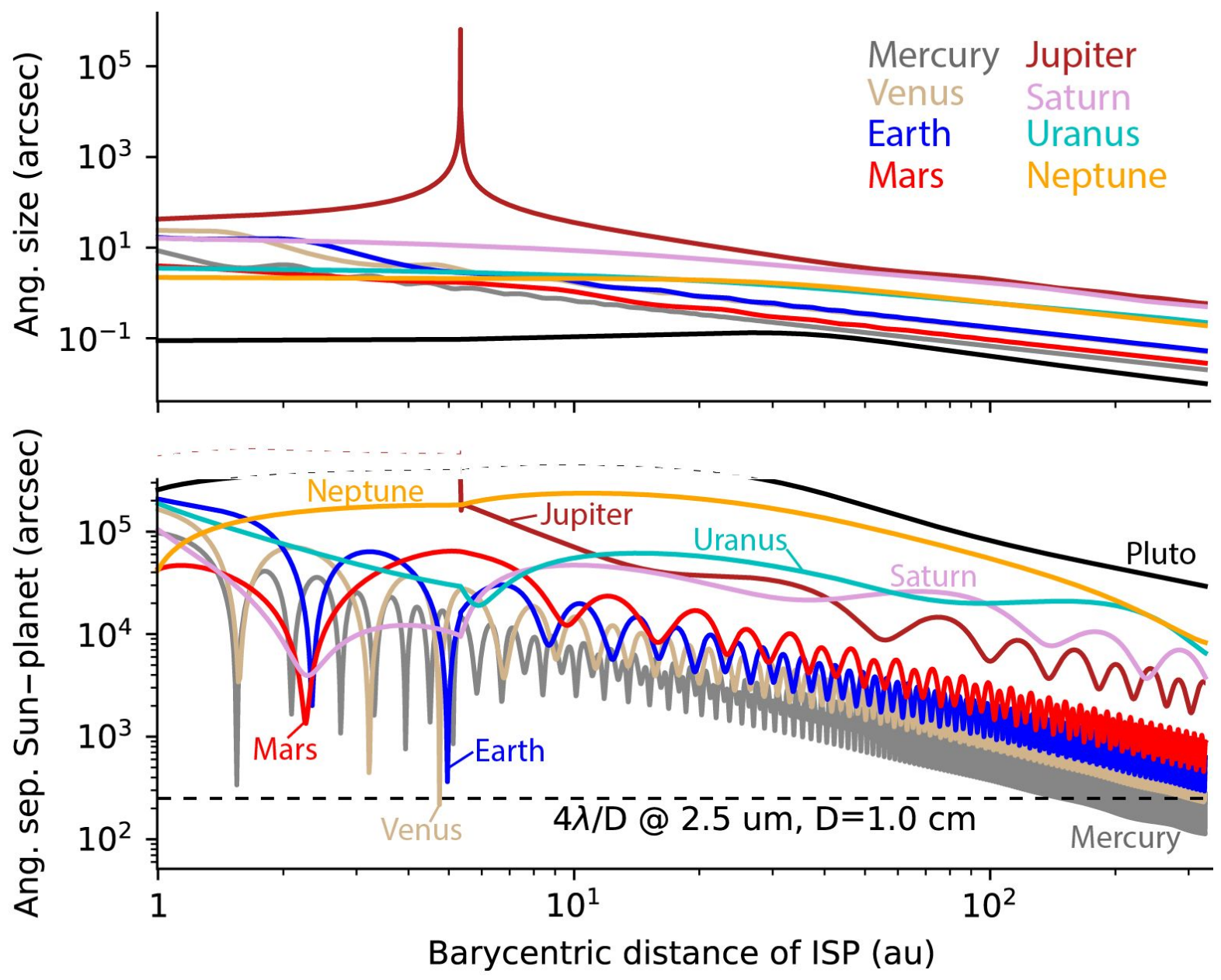

Fig. 1: Launching from Earth in 2030 and using a gravity assist at Jupiter just 10 months later, ISP would fly past Quaoar in 2036, ultimately reaching $\sim 7$ au/year. The top panel shows the angular size of the planets as a function of ISP's position along this course. The bottom panel shows the angular separation between the target planet and the Sun as viewed from ISP. Mercury drops below the diffraction limit (denoted by the black dashed line and $4 \lambda / D$ ) even at maximum separation from the Sun (that is, at quadrature) beyond $\sim 300$ au. [A pinkie held at arm's length is $\sim 1^{\circ}$ or 3600 arcseconds in width, approximately the maximum Jupiter-Sun separation seen from $\sim 300$ au] 


\section{References}

[1] Reuter et al., 2007. Science, 318(5848), pp.223-225; [2] Peplowski et al., 2019. Nature Astronomy, pp.1-4; [3] Grundy et al., 2007. Science, 318(5848), pp.234-237. [4] Robinson, et al., 2014. ApJ, 787(2), p.171; [5] Sagan et al., 1993. Nature, 365(6448), pp.715-721; [6] Christensen \& Pearl, 1997. JGR: Planets, 102(E5), pp.10875-10880. [7] Crow et al., 2011. ApJ, 729(2), p.130; [8] Livengood et al. 2011. Astrobiology, 11(9),p.907; [9] Limaye et al., 2018 SSR, 214(5), p.102; [10] Geissler et al., 1995. JGR: Planets, 100(E8), pp.16895-16906; [11] Marley et al., 2020. Planetary white paper. [12] Brugman et al., 2020. Planetary white paper; [13] Roberge et al., 2012. PASP, 124(918), p.799; [14] Beichman et al., 2019. AGUFM, 2019, pp.SH54A-10.

[15] Mandt et al., 2020. Astro2020 white paper; [16] McNutt et al., 2019. Acta Astronautica, 162, pp.284-299; [17] Schwieterman et al., 2018. Astrobiology, 18(6), pp.663-708; [18] Stephan et al., 2010. JGR, 37, 7; [19] Cowan et al., 2012. ApJL, 752(1), p.L3; [20] Robinson et al., 2010. ApJL, 721(1), p.L67; [21] Lustig-Yaeger et al., 2018. ApJ, 156(6), p.301; [22] Carlson et al., 2019. GRL, 46(17-18), pp.10679-10686; [23] Olson et al., 2018. ApJL, 858(2), p.L14; [24] Mallama \& Hilton, 2018. Astro. \& Comp., 25, pp.10-24; [25] Mallama et al., 2017. Icarus, 282, pp.19-33; [26] Som et al., 2020. Planetary white paper; [27] Hansen \& Hovenier 1974. J. Atmos. Sci. 31 (4), pp.1137-1160; [28] Muñoz et al.. Nature Astronomy, 1(5), pp.1-7; [29] Mayorga et al., 2016 ApJ, 152(6), p.209; [30] Santerne et al., 2018. Nature Astronomy, 2(5), pp.393-400; [31] Lazio et al., 2020. Planetary white paper; [32] Johnson \& Hauck 2016. Elements, 15(1), pp.21-26; [33] Kane et al., 2020. Planetary white paper; [34] Lustig-Yaeger et al., 2020. ApJL. 887(1), L11; [35] Arney et al., 2016. Astrobiology, 16(11), pp.873-899; [36] Ehlmann et al., 2016. JGR: Planets, 121(10), pp.1927-1961; [37] Arridge et al., 2014. PSS, 104, pp.122-140; [38] Krissansen-Totton et al., 2016. ApJ, 817(1), p.31; [39] Sing et al., 2016. Nature, 529(7584), pp.59-62; [40] Gao \& Zhang, 2020. ApJ, 890(2), p.93; [41] Bolcar et al., 2016. ISOP, 9904, p. 99040J; [42] Mennesson et al., 2016, ISOP, 9904, p. 99040L; [43] Santos-Sanz, 2017. EPSC, pp.EPSC2017-97; [44] Shin et al., 2018. ApJ, 863(1), p.23; [45] Harris \& Russo, 2015. Space Policy, 31, pp.1-4; [46] McNutt et al., 2011. Acta Astronautica, 69(9-10), pp.767-776; [47] Runyon et al., 2020. Planetary white paper. 